\title{
Performance Analysis of Using the Next generation Australian SBAS with Precise Point Positioning Capability For Intelligent Transport Systems
}

\author{
Ahmed El-Mowafy \\ School of Earth and Planetary Sciences \\ Curtin University \\ Perth, Australia \\ a.el-mowafy@curtin.ed.au
}

\author{
Norman Cheung \\ HMI Technologies \\ Sydney, Australia \\ norman.cheung@hmitechnologies.com.au
}

\author{
Eldar Rubinov \\ FrontierSI, \\ Melbourne, Australia \\ erubinov@frontiersi.com.au
}

\begin{abstract}
In 2018, a next-generation Satellite-Based Augmentation System (SBAS) test-bed was launched in Australia/New-Zealand in preparation for building an operational system. This new generation SBAS includes L1 legacy SBAS, new dual-frequency multi-constellation (DFMC) SBAS, and orbit and clock corrections for precise point positioning (PPP) using GPS and Galileo. In this paper, the next generation SBAS and its models are first presented, and the benefits of using its new components are discussed. Test results for lane identification applications in Intelligent Transport Systems (ITS) are presented and analyzed. Kinematic tests were performed in different ITS environments. These are characterized by different levels of sky-visibility and multipath, including clear sky, suburban, low-density urban, and high-density urban environments. Performance analysis show that results vary widely depending on the operational conditions but all SBAS solutions have better positioning accuracy compared with the standalone solutions that are currently used in transport applications. The DFMC SBAS slightly outperformed the L1 SBAS, with accuracy at submeter, and it has advantages during periods of fluctuations of the ionosphere with an extended coverage area. As expected, the SBAS-based PPP solutions have shown to give the best positioning precision and accuracy among all tested solution types, with sub-decimeter level accuracy, provided that enough convergence time is available. The paper concluded by giving remarks on the use of this new technology for ITS.
\end{abstract}

Keywords-SBAS, PPP, GPS, Galileo, Intelligent Transport Systems

\section{INTRODUCTION (HEADING 1)}

The Satellite-Based Augmentation System (SBAS) are used in different regions to augment Global Navigation Satellite System observations to improve positioning and navigation of single point positioning (SPP). SBAS uses geostationary (GEO) satellites to transmit orbit and clock corrections, regional ionospheric corrections, ranging and integrity information. Currently operational SBAS includes the USA Wide Area Augmentation System (WAAS), the European Geostationary Navigation Overlay Service (EGNOS), the Japanese Multi-functional Satellite Augmentation System (MSAS), the Russian System for Differential Correction and Monitoring (SDCM) and the Indian GPS-aided GEO Augmented Navigation (GAGAN). These systems, except for MSAS, currently support only single-frequency measurement users but in the future, such as for EGNOS V3 will include DFMC service.

This study is funded through project PD8703 - SBAS for connected vehicles - part of the Australia/NZ SBAS Test-bed project funded by FrontierSI on behalf of Geoscience Australia.
Since September 2017, Australia and New Zealand (referred to from then on as AUS/NZ) commenced SBAS test-bed over the Asia-Pacific and Australasia area [1]. This SBAS comprises a second-generation SBAS service in a pre-step of the building of a fully operational system within a few years. The test-bed provides in addition to the L1 legacy SBAS signals that are transmitted over L1, the dualfrequency multi-constellation (DFMC) SBAS corrections being transmitted over L5. The former (i.e. SBS L1) meets the RTCA/DO-229 standard and is open to current massmarket receivers implementing SBAS positioning mode. The second service; the new generation SBAS L5 DFMC prototype signal, complies with the specification defined in WG62 GAL GPS SBAS MOPS v0.6.1 [2]. The WG62 is a draft standard that defines future DFMC services. Due to bandwidth limitations, the AUS/NZ DFMC service only supports GPS and Galileo satellites for which corrections and integrity data are available [3]. While the SBAS L1 service transmits range, orbit, clock and ionospheric delay corrections, applicable to the service area covering the complete Australian and New Zealand territories, the SBAS L5 DFMC provides GPS and Galileo dual-frequency augmentation that can be used anywhere across the whole footprint of the GEO satellite. For the AUS/NZ SBAS testbed, an Inmarsat 4F1 GEO satellite is used, which is located at longitude of 143.4 degrees East [1].

The AUS/NZ SBAS additionally broadcast precise satellite orbits and clock corrections to support real-time float-ambiguity Precise Point Positioning (PPP) service that can deliver $5-20 \mathrm{~cm}$ accuracy $[4,5,6]$. The corrections are transmitted over L1 frequency for GPS only and L5 for GPS+Galileo. This service allows for superior availability of PPP compared with traditional PPP. It enables PPP anywhere in the footprint of the SBAS GEO satellite, i.e. most Australia-Asia pacific region, whereas traditional PPP depends on the Internet to receive the corrections, and thus is limited to areas where the Internet is available. Noting the vast size of low populated areas in Australia and the surrounding oceans, such service of providing PPP over satellites brings significant commercial benefits.

Preliminary SBAS test-bed messages and positioning results have been reported in $[1,7,8]$. Under good satellite visibility conditions, accuracy at sub-m was obtained when using SBAS L1 and DFMC, and at sub-decimeter using PPP. Whilst the PPP positioning method has higher accuracy than L1 and DFMC SBAS, it requires a period of 
convergence time to achieve this level of accuracy, which can be in the range of 30-60 minutes [9], whereas SBAS L1 and DFMC positioning is achieved almost instantaneously due to being code-based.

To complement the above studies, this paper presents performance analysis of the AUS/NZ second generation SBAS (L1, DFMC) and PPP positioning with a main focus set on its use for advanced transport applications. The outcomes of this research can assist in clarifying the potential of different services of SBAS in improving the safety and efficiency of the road transport in Australia. Since transport applications experience continuous changes in the operation environmental surroundings, the analysis is conducted classifying the work environment into three classes: suburban, low -density urban, and high-density urban. The next section briefly overviews the AUS/NZ SBAS and discuss processing of data. Next, tests are described and results are presented and analysed. Performance metrics such as the obtained precision, accuracy, availability and suitability of positioning solutions for ITS applications are next discussed.

\section{DESCRIPTION OF THE AUS/NZ SBAS}

The second-generation AUS/NZ SBAS test-bed was developed by Geoscience Australia (GA) and Land Information New Zealand (LINZ), and is administrated by the Cooperative Research Centre for Spatial Information (CRCSI) (now known as FrontierSI). The project was contracted to GMV (Spain) in conjunction with Lockheed Martin Space Systems and Inmarsat. The SBAS test-bed aimed to assess the SBAS performance and benefits over a diversity of industries including transport, aviation, mining, agriculture, and maritime applications. The SBAS infrastructure consists of the space segment, the ground segment, and the support segment in addition to the user segment (see Fig 1). The ground segment consists of the CORS network collecting raw GNSS observations, the Augmentation Processing Center (APC) computing the SBAS and PPP corrections, and the uplink system at Uralla (East Australia) that transforms the SBAS messages into electromagnetic signals and sends them to the GEO satellite. The space segment consists of GPS and Galileo satellites, and the GEO satellite Inmarsat-4F1 (PRN 122) that transmits the SBAS signals. The support segment is a magicGNSS Web Monitor at the GMV's premises in Spain which monitors the system and service performance $[7,10]$.

As mentioned earlier, the test-bed continuously broadcasts the following services [1]:

i- SBAS L1 Legacy service: SBAS broadcasts this signal in accordance with RTCA/DO229E [11]. The message broadcasts both corrections and integrity information for GPS satellite ephemeris, and regional ionosphere corrections. Since the service is configured for test purposes only (i.e. not for use in safety applications during the testbed period), it broadcast MT 0 message every 6 seconds. The Test-bed service region is defined to cover the Australia and New Zealand territories as shown in Figure 2 (left panel) where the CORS stations used for generation of the regional ionosphere corrections are located. The ionospheric delay computed at the reference stations is typically given in a grid of $5^{\circ}$ (approximately $500 \mathrm{~km}$ ). The user interpolates these grid values at their location, and computes the slant ionosphere by using a mapping function.

ii. SBAS L5 DFMC service: The Australian SBAS DFMC L5 service is implemented in accordance with WG62 GAL GPS SBAS MOPS v0.3.8_10 draft [3]. The Test-bed signal contains corrections and DFREI bounds applicable to GPS L1/L2 + GAL E1/E5a ionosphere-free combinations. The service coverage area corresponds to the footprint of the PRN 122 GEO as illustrated in Figure 2 (right panel). In addition to the larger coverage area, this service is expected to provide better performance than L1 SBAS during periods of rapid/high fluctuations of the ionosphere.

iii. Real-time PPP (RT-PPP) through SBAS L1 and SBAS L5: The AUS/NZ solution uses the spare bits present in the SBAS message to provide additional information for PPP with a higher resolution. This is compatible with the SBAS service, as the SBAS receivers complying with the standard are instructed to ignore the unused message bits [1]. However, PPP-enabled receivers can use this information to access the high accuracy data. The PPP corrections transmitted within the SBAS L1 provides corrections for GPS L1/L2 signals while the PPP corrections transmitted through the SBAS L5 signal provide corrections for GPS L1/L2 + GAL E1/E5a signals. Both services are available in the entire GEO footprint as shown in Figure 2 (right panel).

In the future operation AUS/NZ SBAS, with enough number of GPS III satellites become available to allow for reliable positioning using L1/L5, DFMC SBAS and RT-PPP will be implemented using the L1/L5 combination instead of $\mathrm{L} 1 / \mathrm{L} 2$ that is used in the test-bed.

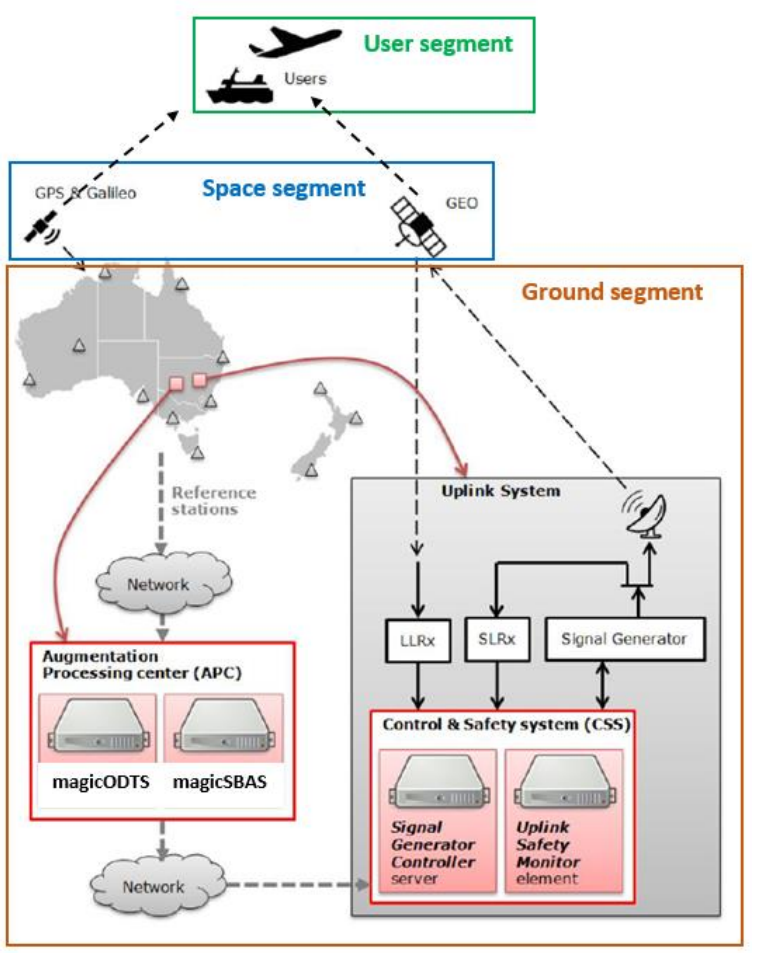

Fig. 1. SBAS test-bed infrastructure 

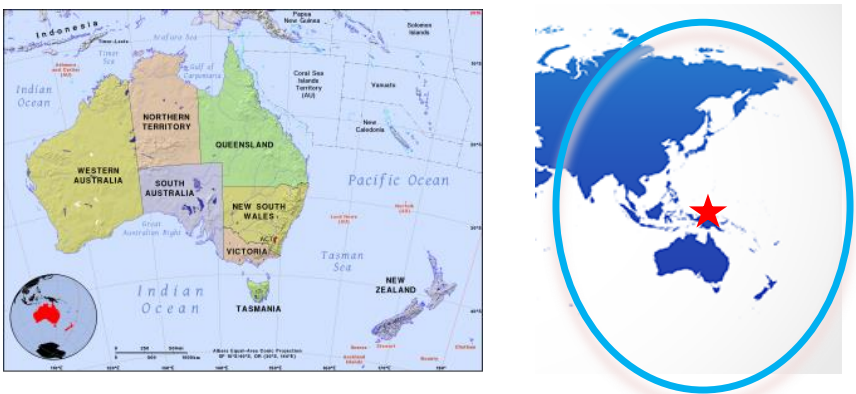

Fig. 2. SBAS L1 (left) and SBAS L5 \& RT-PPP (right) coverage areas

Whilst many off-the-shelf receivers can pick up L1 SBAS, special hardware and software were needed to decode DFMC and PPP messages. In our tests, a Septentrio AsteRx-U receiver and Leica AR10 antenna, with an RF front-end and Linux tablets were used to capture real-time signals and $\log$ data for post processing. The software packages developed by GMV, magicGEMINI, which runs on a Windows platform, was employed for post-processing SBAS data and magicAPK for decoding messages and processing RT-PPP. magicGEMINI accepts raw GNSS observations as well as SBAS messages and outputs SBAS derived positions and corresponding evaluation of the system performance at the user level. On the other hand, magicAPK runs on a Linux platform. Figure 3 depicts a diagram of the hardware and software setup used.



Fig. 3. Hardware and software setup used

\section{SBAS MODELS}

The general GNSS code and phase observations can be expressed as:

$$
\begin{aligned}
P_{j}= & \left\|x^{s}-x_{r}\right\|+c\left(\delta t_{r}-\delta t^{s}\right)+l_{j}+g_{r}^{s} \tau_{r}+m+d_{r}- \\
& d_{s}+e \\
\varphi_{j}= & \left\|x^{s}-x_{r}\right\|+c\left(\delta t_{r}-\delta t^{s}\right)-l_{j}+g_{r}^{s} \tau_{r}+\lambda_{j} a+\mu+ \\
& \delta_{r}-\delta_{s}+\varepsilon
\end{aligned}
$$

where $P_{j}$ and $\varphi_{j}$ are the code and phase observables on the $j$ th frequency (in meters), $x_{s}$ and $x_{r}$ are the positions of satellite and user receiver respectively, $c$ is the speed of light in vacuum, $\delta t_{r}$ and $\delta t_{s}$ are receiver and satellite clock offsets and $l_{j}$ is the slant ionospheric delay. The term $\tau_{r}$ denotes the zenith tropospheric delay (ZTD) with its mapping function $g_{r}^{S}$. $m$ and $\mu$ denote the multipath error on code and phase measurements respectively, $d_{r}, d_{s}$ and $\delta_{r}$, $\delta_{s}$ are the systematic hardware delays, at receiver and satellite, on code and phase measurements. $\lambda_{j}$ is the $j$ th carrier wavelength, $a$ is the carrier phase integer ambiguity in cycles, and finally $e$ and $\varepsilon$ are the random code and phase measurements noise, respectively. The unknowns to be solved for are $x_{r}$ and $\delta t_{r}$. The other terms are sources of error that need to be accounted for to achieve precise positioning.

For the SBAS L1 augmented mode, the used receivers have the option of configuring a carrier phase-based, singlefrequency smoothing filter with individually specified time constants. No smoothing was applied to the measurements at the time of recording. Additionally, a proprietary multipath mitigation technique (APME+, A-Posteriori Multipath Estimator) is applied to improve measurement quality by removing short-delay multipath without introducing biases [1].

In the DFMC solutions, the ionosphere-free combination is formed using the phase-smoothed code observations on GPS L1/L2 and Galileo E1/E5a, denoted as $P_{I F}$ :

$$
\begin{aligned}
P_{I F}= & \frac{f_{i}^{2} P_{i}-f_{j}^{2} P_{j}}{f_{i}^{2}-f_{j}^{2}}=\left|x^{s}-x_{r}\right|+c\left(\delta t_{r}-\delta t^{s}\right)+g_{r}^{s} \tau_{r}+d_{r}- \\
& d^{s}+\varepsilon_{I F}
\end{aligned}
$$

where $f_{i}$ and $f_{j}$ denote the frequencies, and $P_{i}$ and $P_{j}$ denote the smoothed code observations on the $i$ and $j$ frequency, respectively. $\varepsilon_{I F}$ denote the noise, where $I F$ is the ionosphere-free operator. With the satellite orbits and clocks transmitted as SBAS corrections, and the a priori values of $x_{r}$ and $\tau_{r}$ estimated or modelled, the receiver coordinates, receiver clocks and remaining ZTDs are estimated. The receiver hardware biases and the common part of the satellite hardware biases are lumped with the receiver clocks.

The ionosphere-free code range measurements in the DFMC SBAS are smoothed by carrier phase measurements with a Hatch filter that uses a fixed time constant of 100 seconds and a weighting factor $(\alpha)$ as described in RTCA/DO-253 [12]. The smoothed code observations for the two frequencies $i$ and $j$ are expressed as:

$$
\begin{gathered}
P_{I F}^{\prime}=P_{I F-1}+\frac{\lambda_{i}\left(\varphi_{i_{k}}-\varphi_{i_{k-1}}\right)-\gamma \lambda_{j}\left(\varphi_{j_{k}}-\varphi_{j_{k-1}}\right)}{1-\gamma} \\
P_{I F k}=\alpha\left(\frac{P_{i_{k}}-\gamma P_{j_{k}}}{1-\gamma}\right)+(1-\alpha) P_{I F}^{\prime}
\end{gathered}
$$

where $P_{I F}$ is the ionosphere-free dual-frequency carriersmoothed pseudorange at the epoch $k, P_{i_{k}}$ and $P_{j_{k}}$ are the raw pseudorange for frequencies $i$ and $j, \varphi_{i_{k}}$ and $\varphi_{j_{k}}$ are the carrier phase measurement for the two frequencies in cycles at $k, \gamma$ is a frequency ratio, which is $(154 / 115)^{2}$ for Galileo measurements on E1 and E5a, and $(154 / 120)^{2}$ for GPS measurements on L1 to L2. In the first 100 seconds since filter initialization, $\alpha$ is equal to the sample interval divided by the time since filter initialization; after 100 seconds $\alpha$ is equal to the sample interval divided by 100 seconds [1]. Newer versions of the DFMC MOPS suggest an increase in the 100 seconds time constant since the code-carrier divergence effect is not a worry for the smoothed ionosphere-free code observations. 
For the PPP solutions, the ionosphere-free phase observations were used in addition to the code observations. The ambiguity parameters and the ZTDs were modelled as random walk bias parameters. [4, 13 and 6] describe the models used in real-time PPP processing.

\section{TEST DESCRIPTION}

Testing of the second generation SBAS in this study was conducted with focus on two focus areas: $i$ 'Heavy Vehicle Efficiency' to examine the SBAS-based positioning ability to improve transport network efficiency by identifying which lane the vehicle is travelling in, and ii. 'Road Safety' to examine the ability of SBAS in supporting issuing collision alerts to drivers. The tests were carried out in July and August 2018 in Wollongong and Sydney, Australia. All data were collected and processed at a frequency of $1 \mathrm{~Hz}$. One of the vehicles used in these tests is displayed in Figure 3 , showing the SBAS antennae mounted on the roof.
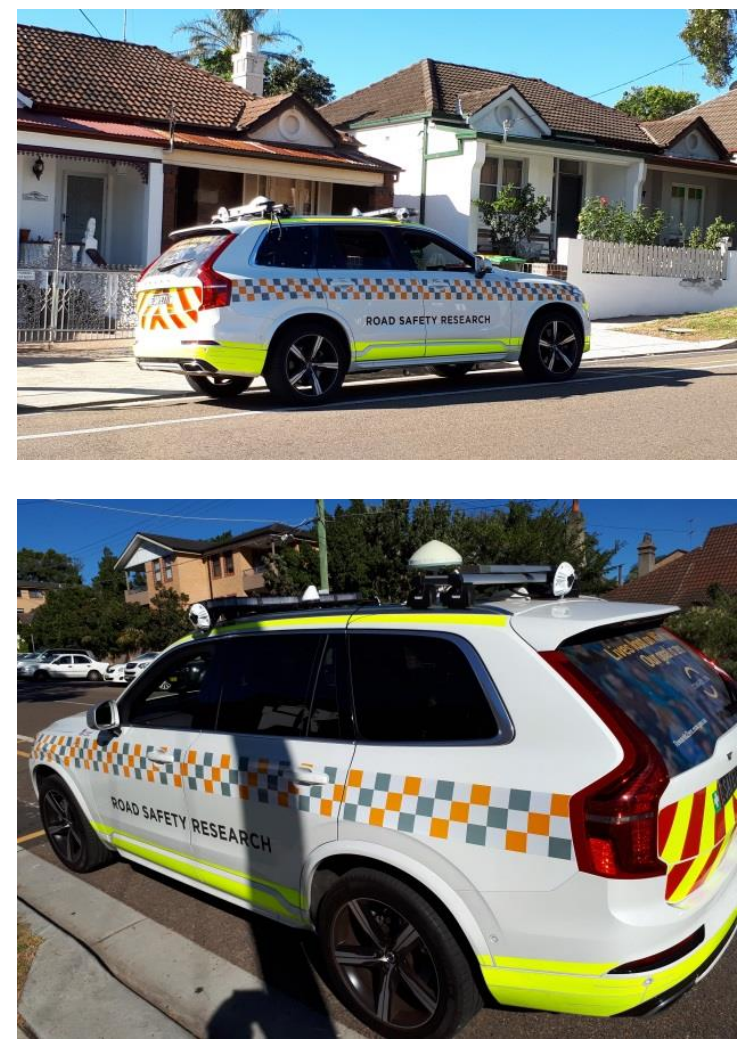

Fig. 3. One Test vehicle

As mentioned earlier, it was observed that both SBAS and PPP positioning performance is heavily dependent on the environment of application, which varies considerably in the transport sector. Therefore, the test environment was subdivided into the following three categories:

- Suburban: characterised by low-rise buildings, with a maximum of three floors,

- Low-density urban: with the presence of low-rise and some high-rise buildings on one side of the road,

- High-density urban: characterised by high-rise buildings on most sides of the road.

An example of one test run that includes all these environments is illustrated in Figure 4.
To evaluate the accuracy of L1 and DFMC SBAS and PPP positioning results, a 'ground truth' for the positions of the vehicle was computed in post-processing mode through independent relative kinematic positioning (PPK). The same raw code and phase observations used for SBAS-based positioning were utilized with data from Continuously Operating Reference Stations (CORS), serving as base stations, where the test vehicles were within a radius of eight kilometers from the base stations. Only ambiguityfixed solutions from PPK, with $1-5 \mathrm{~cm}$ precision, were used as ground truth.

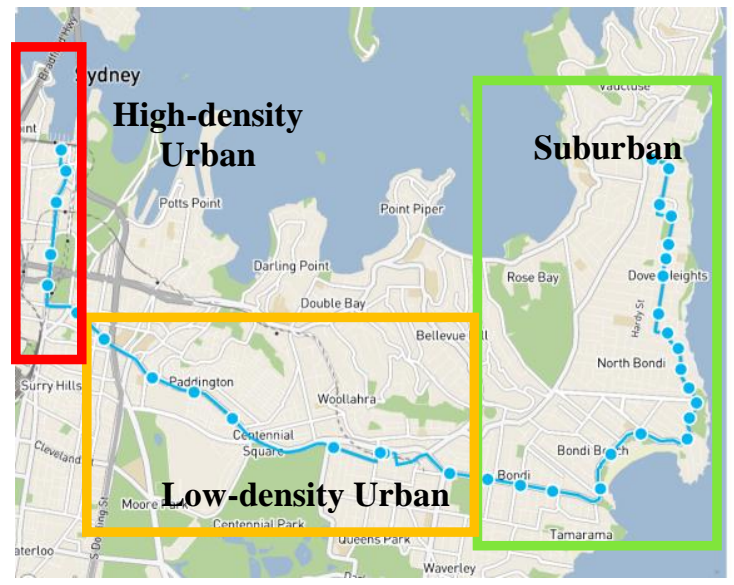

Fig. 4: Test route with subdivision in environments

\section{POSITIONING RESULTS}

Restricting our focus on transport, where horizontal positioning is of interest, test results are illustrated in terms of the time series of the horizontal positioning errors in North and East directions for the different SBAS positioning modes for selected representative examples of the tests that were carried out in this experiment. In addition, the Horizontal Dilution of Precision (HDOP) is depicted, which signifies the number and geometry of observed satellites. The performance indicators considered are availability, mean error and root mean square error (RMSE), where:

- Availability is the fraction of time in which a position solution was delivered over the total time.

- Mean Error and RMSE were computed after all outliers were removed. The mean error is a measure of any bias possibly affecting positioning, whereas the RMSE is a measure of the positioning accuracy.

\section{A. Suburban environment}

Figure 5 to Figure 7 depict the L1, DFMC SBAS and real-time PPP North and East positioning errors respectively for one example test in the suburban environment. The gaps seen for some positioning modes refer to unavailable positions due to observing a limited number of satellites resulting from signal obstruction by structures or trees. 


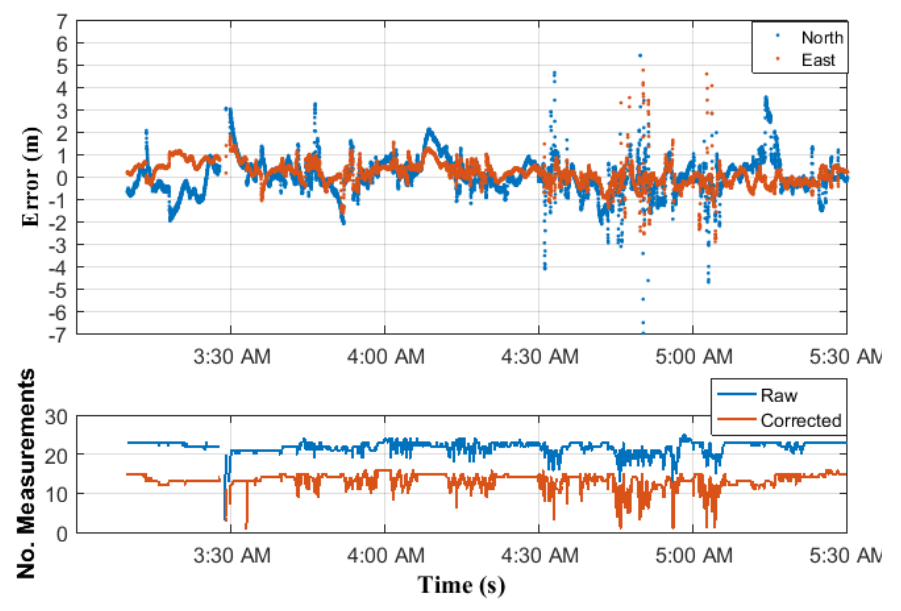

Fig. 5. SBAS L1 results, suburban environment.
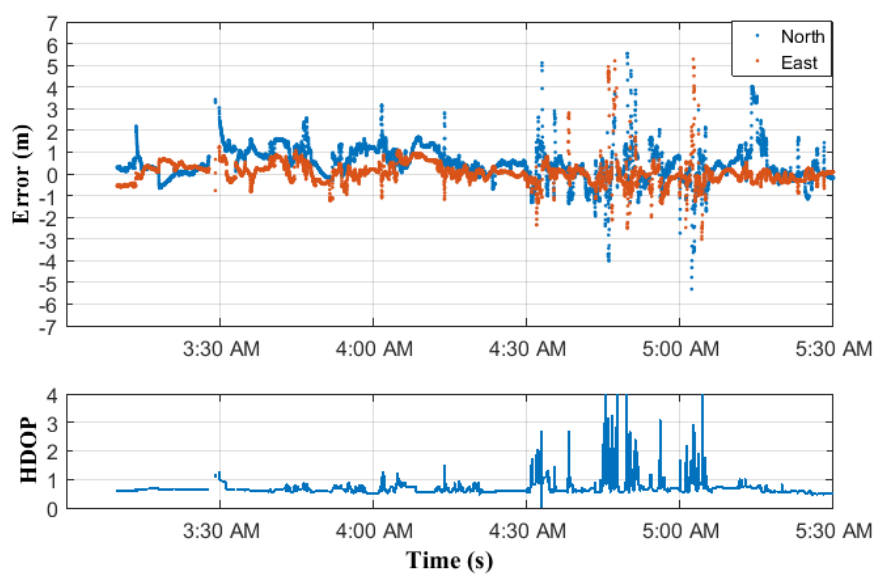

Fig. 6. DFMC results, suburban environment.

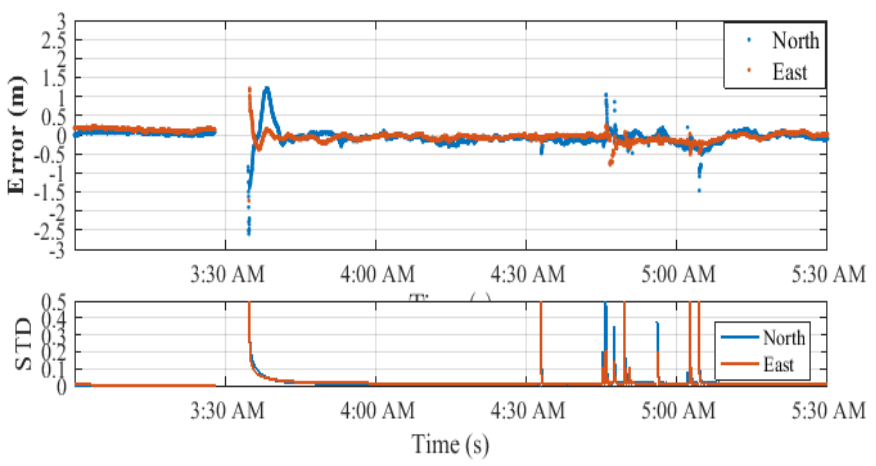

Fig. 7. PPP results, suburban environment

From the Figures 5 to 7, one can see that the positioning performance as expected is highly correlated with HDOP. For real-time PPP, the figures show that the loss of visibility of some satellites led to a temporary/partial loss of convergence, and some time was needed to re-converge to the previous precision levels. The average statistics for the full day of testing are given in Table I, listing also for comparison purpose the results of the traditional Single Point Positioning (SPP). The table shows positioning availability, mean error and RMSE. In addition, Figure 8 shows the mean absolute 2D horizontal error for the different positioning modes, which demonstrates average positioning errors and hence the system accuracy, regardless of the sign of the errors. It can be seen that both L1 and
DFMC SBAS gave much better positioning accuracy compared with SPP, and thus they can provide sub-meter horizontal positioning accuracy for road applications when sufficient number of satellites can be observed. The table and figure 8 show that the overall performance in terms of availability and accuracy of DFMC SBAS and L1 SBAS is close, with some improvement in the former compared with the latter. This is due to the fact that the latter approach uses interpolated values for the ionosphere delay (introducing some interpolation errors), while the former removed the most significant first-order ionosphere delay, at the expense of increased observation noise, through the use of the ionosphere-free combination. This trade-off resulted sometimes in one method giving slightly better results than the other.

Naturally, PPP after solution convergence, and since it relays on the more precise carrier-phase observations, outperformed the L1 and DFMC SBAS methods, which use carrier phase smoothed-code observations, in terms of all studied metrics, with $0.2 \mathrm{~m}$ PPP average accuracy. The suitability of the obtained performance and accuracy for lane level identification, which is a main objective of using SBAS positioning for ITS applications is discussed in the next section.

TABLE I - STATISTICS FOR TESTING IN SUBURBAN ENVIRONMENT

\begin{tabular}{|l|l|l|l|l|}
\hline KPI & SPP & SBAS LI & DFMC & $\boldsymbol{P P P}$ \\
\hline Availability & 0.96 & 0.99 & 0.99 & 1.00 \\
\hline Mean error North (m) & -0.90 & 0.00 & -0.19 & -0.05 \\
\hline Mean error East (m) & -0.08 & 0.15 & 0.04 & -0.03 \\
\hline Mean error Up (m) & -1.66 & -1.32 & -0.45 & -0.11 \\
\hline RMSE North (m) & 1.48 & 0.68 & 0.57 & 0.20 \\
\hline RMSE East (m) & 0.78 & 0.50 & 0.32 & 0.13 \\
\hline RMSE Up (m) & 2.69 & 1.75 & 1.24 & 0.39 \\
\hline
\end{tabular}

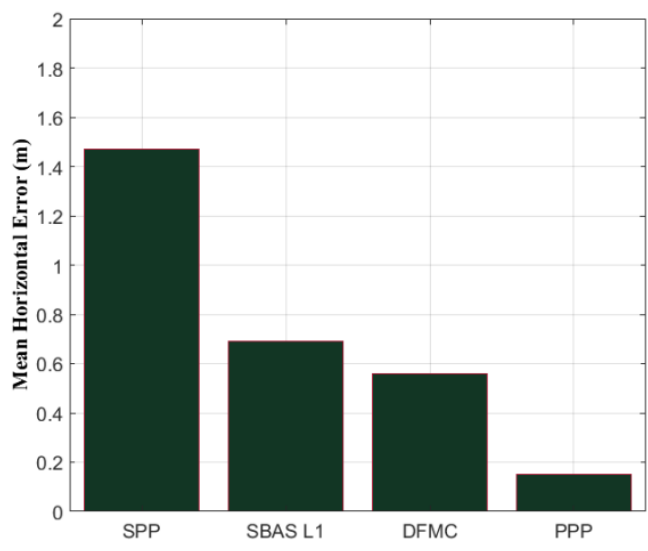

Fig. 8. Overall mean horizontal error for suburban test

\section{B. Low-density and High-density urban environments}

Figure 9 and Figure 11 show the time series of the positioning error versus HDOP for DFMC SBAS and PPP when testing the new generation SBAS in the low-density urban environment. For the high-density urban areas, the availability of positioning was below $30 \%$ for all 
positioning methods due to the obstruction of the signals, of either satellite observations or SBAS signals, or both, by the surrounding buildings. Therefore, the plots for this scenario are not shown due to the low number of positions available. However, the descriptive statistics for the average positioning errors in both the low-density and high-density urban areas are given in Tables II and III, respectively.
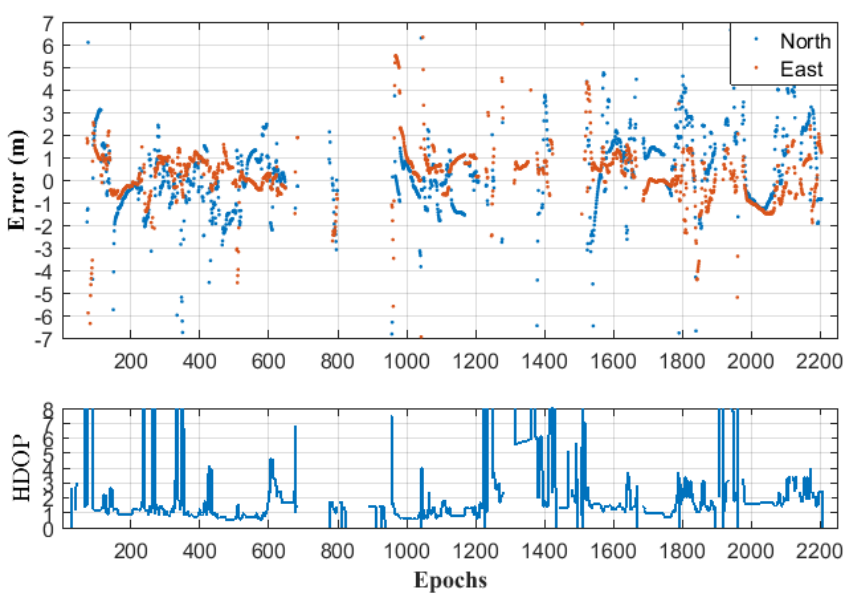

Fig. 9. DFMC results, low-density urban environment.
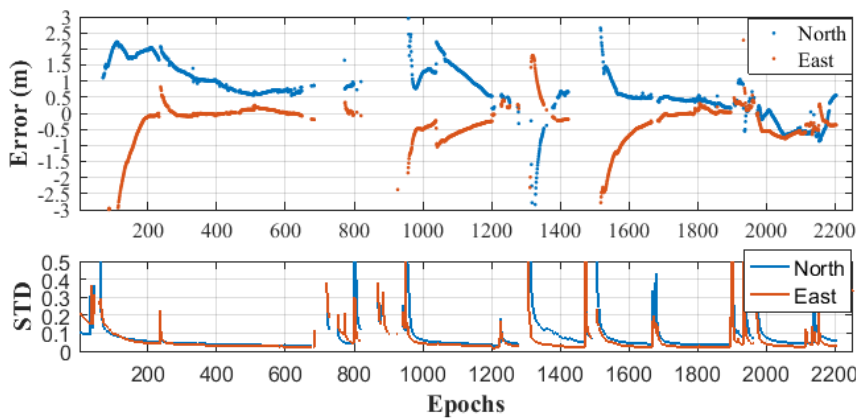

Fig. 10. PPP results low-density urban environment

TABLE II - STATISTICS FOR LOW-DENSITY URBAN ENVIRONMENT

\begin{tabular}{|l|l|l|l|l|}
\hline KPI & SPP & SBAS L1 & DFMC & $\boldsymbol{P P P}$ \\
\hline Availability & 0.67 & 0.83 & 0.83 & 0.91 \\
\hline Mean error North (m) & 0.98 & 0.46 & 0.34 & 0.67 \\
\hline Mean error East (m) & 0.75 & 0.40 & 0.20 & -0.26 \\
\hline Mean error Up (m) & 0.43 & -0.68 & 0.60 & -0.06 \\
\hline RMSE North (m) & 2.38 & 1.71 & 1.75 & 0.99 \\
\hline RMSE East (m) & 1.42 & 1.17 & 0.98 & 0.56 \\
\hline RMSE Up (m) & 3.16 & 2.99 & 2.90 & 1.41 \\
\hline
\end{tabular}

As the figures 9 and 10 show, the HDOP in the urban environment was much higher compared to that in the suburban environment, due to observing less number of satellites with poorer geometry, which affected both positioning accuracy and availability (with some scattered positioning results). For the PPP, the solution had to reinitialize more often. Furthermore, it can be seen from the tables how the performance of both SBAS and PPP methods degraded by the urban environment. For instance, availability of positioning is lower compared to suburban environment, which is a common problem for all GNSS positioning methods. Yet again, a slight improvement of the DFMC mode over the L1 SBAS can be seen.

TABLE III - STATISTICS FOR HIGH-DENSITY URBAN ENVIRONMENT

\begin{tabular}{|l|l|l|l|l|}
\hline KPI & SPP & SBAS L1 & DFMC & PPP \\
\hline Availability & 0.11 & 0.29 & 0.29 & 0.21 \\
\hline Mean error North (m) & -0.60 & 2.90 & -0.22 & 0.23 \\
\hline Mean error East (m) & 1.22 & -0.19 & -1.73 & 0.51 \\
\hline Mean error Up (m) & -9.05 & -6.74 & -4.71 & 1.37 \\
\hline RMSE North (m) & 1.95 & 4.40 & 3.23 & 0.27 \\
\hline RMSE East (m) & 1.26 & 1.15 & 2.66 & 0.71 \\
\hline RMSE Up (m) & 9.08 & 8.01 & 5.68 & 1.65 \\
\hline
\end{tabular}

In the less dense urban environments, the availability for the SBAS modes was over $85 \%$ and over $90 \%$ for PPP. Accuracy was in the order of $2 \mathrm{~m}$ along North and East directions for L1 and DFMC SBAS, and it was $0.5 \mathrm{~m}$ to $1 \mathrm{~m}$ for PPP (highly varying in time due to losses of lock causing re-initialization of convergence), and the rate of outliers was still high $(>5 \%)$ for all positioning modes. On the other hand, in the suburban environments with good sky visibility, the availability of SBAS modes and PPP was over $98 \%$ and the outlier's rate was reasonably low. The accuracy obtained was about $1 \mathrm{~m}$ along North and East for SBAS and $0.1 \mathrm{~m}$ to $0.4 \mathrm{~m}$ for PPP.

Results show that in the high-density urban environment the obtained accuracy when positioning was available was in the order of $2 \mathrm{~m}$ to $3 \mathrm{~m}$ for L1 and DFMC SBAS, and it was better using PPP at $1 \mathrm{~m}$ to $2 \mathrm{~m}$, noting here that the solution convergence was not reached. The degradation of accuracy is also attributed to the presence of multipath and No Line of Sight signals (NLOS). Moreover, the poor satellite availability led to high rate of outliers. Therefore, SBAS-based positioning, and GNSS positioning in general, cannot be solely used for transport applications in the urban environment as well known, and they should be supplemented by other positioning sensors such as Inertial Measurement units (IMU), cameras, radar and LiDAR.

\section{Two vehicles results}

In transport, one should not only consider the absolute positions of vehicles, but also their relative positions with respect to other surrounding vehicles sharing the road. Figure 11 shows a representative example of results of two vehicles in the top two panels of the figure when driving side-by-side in two adjacent lanes in a suburban environment test. The figure depicts the DFMC SBAS North-East horizontal errors as an example. The bottom panel shows the difference between the errors of the two vehicles.

A significant correlation between the results of the two vehicles can be seen. This results in the difference between them having a smaller dispersion, comparable to the dispersion of errors for each vehicle independently. The cross-correlation between the errors of the two vehicles for different runs was from 0.35 to 0.55 for both L1 and DFMC SBAS and PPP. The presence of a positive cross-correlation 
is desirable, as it could be exploited for relative positioning and integrity checks in Vehicle to Vehicle (V2V) localization.
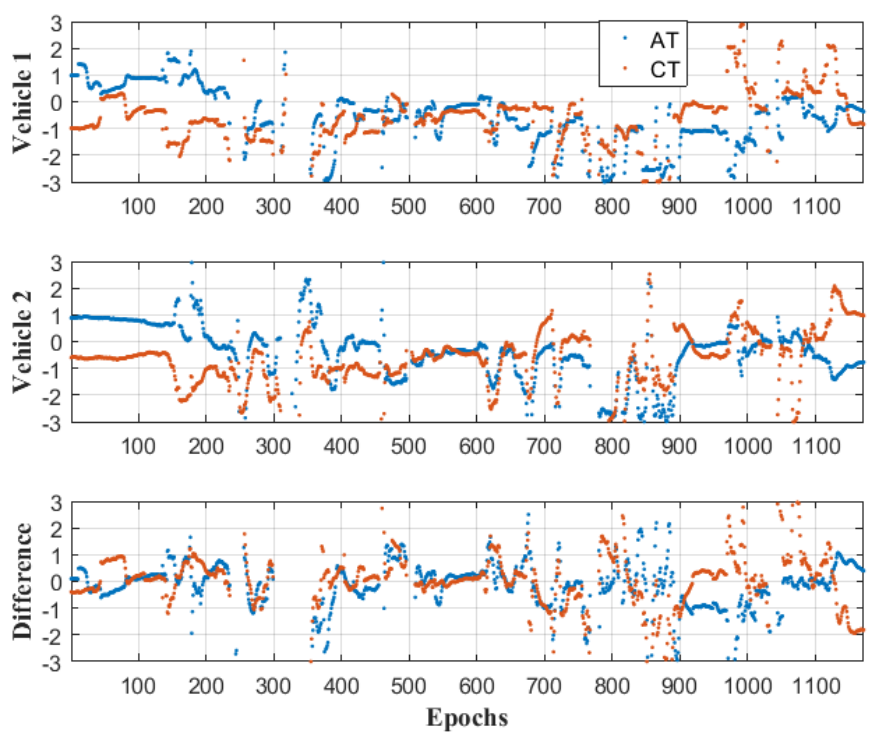

Fig. 11. North-East errors in SBAS DFMC positioning for the two vehicles (top two panels) and their difference (bottom panel) - units are in (m)

\section{SBAS FOR INTELLIGENT TRANSPORT SYSTEMS}

In addition to vehicle localization, a primary use of SBAS in intelligent transport systems is for lane identification, i.e. defining the lane in which the vehicle is located in. This is important for applications such as tolling, traffic light signal control with truck priority, V2V, etc. In Australia, lane width can vary between $2.7 \mathrm{~m}$ and $3.5 \mathrm{~m}$. Thus, assuming a lane width of $2.9 \mathrm{~m}$ and a vehicle width of $2 \mathrm{~m}$ as illustrated in Figure 12 and assuming the GNSS antenna is located in the center of the vehicle in the cross direction, and for the longitudinal direction it is typically located in the rear third of the vehicle. The vehicle can be considered located in a certain lane when its position lies within an allowable range ( $\mu$ ) from the centerline of the lane.
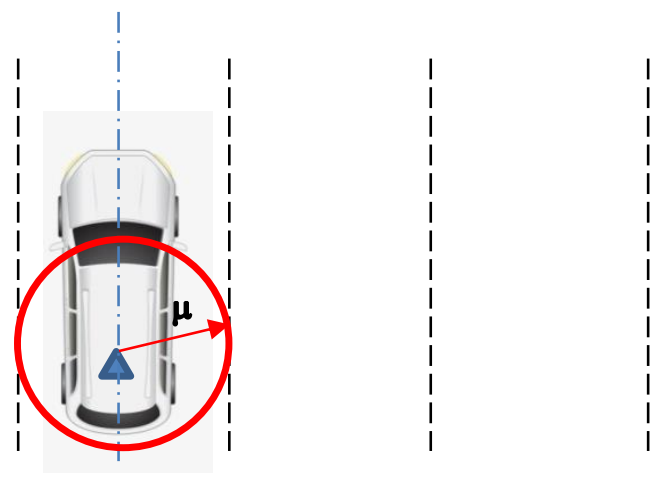

Fig. 12. Illustration of vehicle lane identification

One approach here is to set the acceptable range $\mu$ at less than half of the assumed lane width, i.e. $<1.45 \mathrm{~m}$, such that the majority of the vehicle width is kept in the lane. Positions collected over a few epochs can also help in validating the lane in which the vehicle is travelling in, i.e. when consecutive positions indicate that the vehicle is travelling in the same lane. Let us assume that the threshold for the position error $(\mu)$ is set to $1.4 \mathrm{~m}$, and let us denote the absolute horizontal mean error as $d \bar{H}$, the criterion to identify that the vehicle is in a specific lane can be expressed as:

$$
d \bar{H} \leq \mu \text {, i.e. } d \bar{H} \leq 1.4 \mathrm{~m}
$$

recalling that $d \bar{H}$ is estimated in our tests as the difference between the SBAS-based positions and the reference trajectory. However, one should note that since the mean results are used, the test addresses a $50 \%$ probability. Results of this check based on outcomes from previous tables are summarized in Table IV. In summary, it is concluded that the use of SPP was not acceptable in all cases, whereas SBAS for both L1 and DFMC was mostly acceptable only in the open sky environment, where the geometry and number of satellites was good. PPP was acceptable when the number of visible satellites permitted convergence of the solution.

Table IV: TESTING THAT THE VEHICLE IS IN A SPECIFIC LANE (AVERAGE OVERALL CONCLUSION)

\begin{tabular}{|l|c|c|c|c|}
\hline Environment & SPP & $\begin{array}{c}\text { SBAS L1 } \\
\text { (enabled) }\end{array}$ & $\begin{array}{c}\text { SBAS } \\
\text { DFMC } \\
\text { (enabled) }\end{array}$ & PPP \\
\hline Suburban & Fail & Pass & Pass & Pass \\
\hline $\begin{array}{c}\text { Low-density } \\
\text { urban }\end{array}$ & Fail & Fail & Fail & Pass \\
\hline $\begin{array}{c}\text { High-density } \\
\text { urban }\end{array}$ & Fail & Fail & Fail & Fail \\
\hline
\end{tabular}

The rate of false collision alert was also evaluated. Occurrence of a collision alert was linked to the estimated relative position between the two vehicles such that when the estimated distance between the two vehicles exceeds a safety threshold, it is assumed a warning is raised. The thresholds were selected at $4.5 \mathrm{~m}$ and $2.5 \mathrm{~m}$ for the AlongTrack (direction of motion) and Cross-Track directions, respectively. The values of the threshold were chosen based on the assumptions that the average length of the test vehicles is $3.5 \mathrm{~m}$ (allowing for an along-track separation of $1 \mathrm{~m}$ between vehicles); average width of vehicles is $2 \mathrm{~m}$ (allowing for a cross-track separation of $0.5 \mathrm{~m}$ ). Results show that false collision alerts, when defined with the small margins above, were quite rare, except for the scenarios with vehicles travelling in adjacent lanes, where they can reach $5 \%$ for L1 and DFMC SBAS modes, but still $<1 \%$ for PPP. In all other scenarios the false alert rate was lower than $1 \%$.

One should note that the float-ambiguity PPP solution as implemented in this study suffers from the long time needed for solution convergence. This can be acceptable for long routes such as highways, where signal interruptions and the need to re-initialize PPP are minimal. In other situations, it is recommended that the use of PPP with ambiguity resolution (PPP-AR) be considered in the future, supplemented by the ionosphere information computed in SBAS, since this method offers a shorter convergence time and possibly better accuracy [14]. 


\section{CONCLUSION}

Testing show that SBAS positioning in open sky environment and when observing satellites with good number and geometry can reach horizontal accuracy in the order of sub-meter. This level of accuracy under such conditions is sufficient for localization of vehicles within a lane on the transport network for use in ITS applications, which is not possible to be reliably achieved with existing GNSS SPP methods. The use of DFMC SBAS using GPS+Galileo slightly improves positioning accuracy compared to the legacy L1 SBAS. The DFMC SBAS also provides extended coverage area and can provide better performance during periods of high fluctuations of the ionosphere. Providing orbit and clock corrections for PPP through SBAS satellites is advantageous in terms of enabling a wide area of coverage without the dependence on availability of the Internet, which is of particular interest to the vast remote areas in Australia. While PPP is able to provide positioning with accuracy $<0.2 \mathrm{~m}$, it requires approximately 30 minutes or more for solution convergence at start up or after visibility is lost for extended periods. This need for convergence makes the PPP solution less appealing for short journeys but it can be used for long trips, e.g. along highways.

While accuracy of SBAS-based positioning in suburban environment can reach sub-meter accuracy, this accuracy deteriorates in urban environments, reaching a couple of meters, mainly owing to poor satellite geometry, multipath and NLOS. These are common concerns in positioning using GNSS. Therefore, positioning by GNSS in the urban environment should be supplemented with other positioning sensors such as IMU, cameras, radars and LiDAR.

\section{ACKNOWLEDGEMENTS}

The authors would like to sincerely thank FrontierSI, and Geoscience Australia (GA) for their support during this study. Dr Davide Imparato is acknowledged for his role in data processing. Transport for New South Wales and Dr Joon Wayn Cheong from the University of New South Wales are acknowledged for their help in data collection.

\section{REFERENCES}

[1] H.Sobreira, B.Bougard, J.Barrios, J.D. Calle, "SBAS Australian-NZ Test Bed: Exploring New Services", Multi GNSS Asia Conference. Melbourne, Australia. 23-25 October 2018.

[2] Minimum Operational Performance Specification for Galileo / Global Positioning System /Satellite-Based Augmentation System Airborne Equipment; SBAS DFMC L5 MOPS Draft WG62_GAL_GPS_SBAS_MOPS_v0.6.1_21_Jun_2018.

[3] Minimum Operational Performance Specification for Galileo / Global Positioning System /Satellite-Based Augmentation System Airborne Equipment; SBAS DFMC L5 MOPS Draft WG62_GAL_GPS_SBAS_MOPS_v0.3.8_10_Mar_2017.

[4] J.F. Zumberge, M.B. Heftin, D.C. Jefferson, M.M. Watkins, and F.H Webb. "PPP for the efficient and robust analysis of GPS data from large networks", J Geo.Res., vol 102, no. B3, pp. 5005-5017, 1997.

[5] P.Heroux, Y. Gao, J. Kouba, F. Lahaye, Y. Mireault, P. Collins, K. Macleod, P. Tetreault, K. Chen, "Products and Applications for Precise Point Positioning - Moving Towards Real-Time, Proc. of the
ITM of the ION GNSS, Long Beach, CA, pp 1832 - 1843, Sept 21 24, 2004.

[6] A. El-Mowafy, M. Deo, N. Kubo, "Maintaining real-time precise point positioning during outages of orbit and clock corrections" GPS Solutions, vol. 21, no. 3, pp. 937-947, 2017.

[7] J. Barrios, J. Caro, J.D. Calle, E. Carbonell, J.G. Pericacho, G.Fernández, V.M. Esteban, M.A. Fernández, F. Bravo, B. Torres, A. Calabrese, A. Diaz, I. Rodríguez, M.D. Laínez, M.M. Romay, R. Jackson, P.E. Reddan, D Bunce, C. Soddu, "Update on Australia and New Zealand DFMC SBAS and PPP System Results". In proceedings of ION GNSS+ 2018, Miami, Florida, September 24-28, 2018, pp. 1038-1067

[8] K. Cogdell, P. Reddan, “ Australia/New Zealand DFMC SBAS and Navigation Message Authentication". In proceedings of ION GNSS+ 2018, Miami, Florida, September 24-28, 2018, pp. 1068-1083

[9] A. El-Mowafy, "Real-Time Precise Point Positioning Using Orbit and Clock Corrections as Quasi-Observations for Improved Detection of Faults.” J. of Navigation, vol. 71, no. 4, pp.769-787, 2018.

[10] Technical Specifications Technical Specifications Document for Satellite-Based Augmentation System (SBAS) Testbed. Revision 5, https://www.crcsi.com.au/assets/Program-1/SBAS-Project/AustraliaNZ-Testbed-Technical-Specifications-Rev05.pdf.,10 November 2017.

[11] RTCA, DO-229E Minimum Operational Performance Standards for Global Positioning System Airborne Equipment, 2016.

[12] RTCA, DO-253D Minimum Operational Performance Standards for GPS Local Area Augmentation System Airborne Equipment, 2017.

[13] J. Kouba, "A Guide to Using International GNSS Service (IGS) Products" Geodetic Survey Division, Natural Resources Canada, http://acc.igs.org/UsingIGSProductsVer21.pdf, pp, 1-32, ,May 2009,

[14] M. Deo and A. El-Mowafy "A method for Precise Point Positioning with Integer Ambiguity Resolution with Triple-frequency GNSS Data", Measurement Science and Technology, In Press. 